DOE/ER/40417-48

\title{
NUCLEAR STRUCTURE RESEARCH
}

Progress Report for the Period

August 1, 1993 - July 31, 1994

\author{
Daeg S. Brenner \\ Clark University \\ Worcester, MA 01610
}

\author{
August 1994 \\ Prepared for \\ The U.S. Department of Energy \\ Under Grant No. DE-FG02-88ER40417
}

NOTICE

This report was prepared as an account of work sponsored by the United States Government. Neither the United States nor the United States Department of Energy, nor any of their employees, nor any of their contractors, subcontractors, or their employees, makes any warranty, express or implied, or assumes any legal liability or responsibility for the accuracy, completeness, or usefulness of any information, apparatus, product or process disclosed or represents that its use would not infringe privately owned rights. 


\section{DISCLAIMER}

\section{Portions of this document may be illegible in electronic image products. Images are produced from the best available original document.}




\section{TABLE OF CONTENTS}

Page

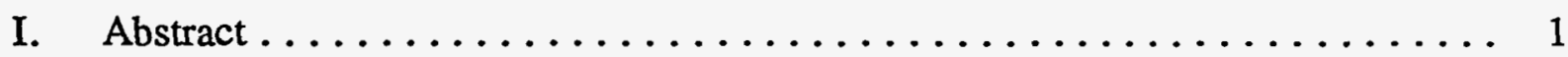

II. Nuclear Structure Research $\ldots \ldots \ldots \ldots \ldots \ldots \ldots \ldots \ldots \ldots \ldots \ldots \ldots$

A. Introduction $\ldots \ldots \ldots \ldots \ldots \ldots \ldots \ldots \ldots \ldots \ldots \ldots \ldots \ldots \ldots \ldots \ldots$

B. Publications $\ldots \ldots \ldots \ldots \ldots \ldots \ldots \ldots \ldots \ldots \ldots \ldots \ldots \ldots \ldots \ldots \ldots$

1. Letters ............................. 3

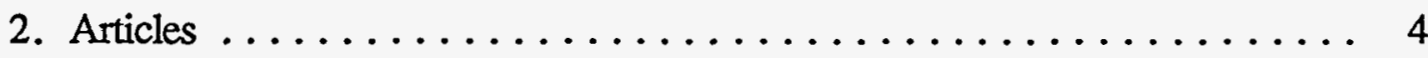

3. Conference Proceedings $\ldots \ldots \ldots \ldots \ldots \ldots \ldots \ldots \ldots \ldots \ldots \ldots \ldots \ldots \ldots \ldots$

C. Professional Activities $\ldots \ldots \ldots \ldots \ldots \ldots \ldots \ldots \ldots \ldots \ldots \ldots$

1. Contributed Papers/Abstracts/Posters $\ldots \ldots \ldots \ldots \ldots \ldots \ldots \ldots \ldots$

2. Invited Talks $\ldots \ldots \ldots \ldots \ldots \ldots \ldots \ldots \ldots \ldots \ldots \ldots \ldots \ldots \ldots \ldots$

3. Education $\ldots \ldots \ldots \ldots \ldots \ldots \ldots \ldots \ldots \ldots \ldots \ldots \ldots \ldots \ldots \ldots \ldots \ldots$

4. Professional Service $\ldots \ldots \ldots \ldots \ldots \ldots \ldots \ldots \ldots \ldots \ldots$

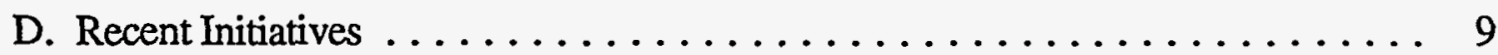

1. g-Factor of the $3 / 2+121.8 \mathrm{keV}$ Level in $99 \mathrm{Zr} \ldots \ldots \ldots \ldots \ldots \ldots$

2. Evidence for a Spherical Subshell at $\mathrm{N}=164 \ldots \ldots \ldots \ldots \ldots \ldots$

3. The Phonon Structure of $122 \mathrm{Cd} \ldots \ldots \ldots \ldots \ldots \ldots \ldots \ldots \ldots$

4. Mass and Decay of the $\mathrm{N}=\mathrm{Z}$ Nuclide $68 \mathrm{Se} \ldots \ldots \ldots \ldots \ldots \ldots \ldots$

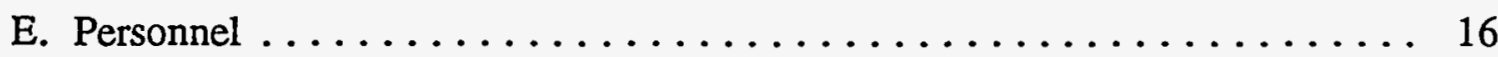

III. Acknowledgement ......................... 17 


\section{ABSTRACT}

The Clark University research program in nuclear structure is a collaborative effort involving Clark University personnel, staff members from Brookhaven National Laboratory and an active participation of scientists from the U.S. and other nations. The TRISTAN on-line isotope separator and the capture $\gamma$-ray facility at the High Flux Beam Reactor have been the primary experimental foci of the program although experiments have been carried out at other installations such as the High Flux Reactor of the Institute Laue-Langevin in Grenoble and at the Tandem Van de Graaff Accelerator, Yale University. Recently, we have joined colleagues from Argonne National Laboratory, Vanderbilt University and the University of Maryland in experiments using the Fragment Mass Analyzer (FMA) at the ATLAS accelerator.

Our program has four principal research themes which underlie the various research initiatives, three involving nuclear structure physics and one directed towards astrophysics. These themes are: (1) the critical role of the proton-neutron interaction in the evolution of nuclear structure and its relation to collectivity, (2) the study of exotic nuclei far-from-stability produced by radioactive ion beams (RIBs), (3) the appearance and role of dynamical symmetries in nuclei and the characterization of transitions among these limits, and (4) the characterization and prediction of properties of nuclei far from stability of importance in stellar nucleosynthesis.

The activities involving Clark personnel during the operating period of Grant No. DE-FG02-88ER40417, August 1, 1993 - July 31, 1994, are summarized below.

\section{DISCLAIMER}




\section{NUCLEAR STRUCTURE RESEARCH}

\section{A. Introduction}

The Clark University nuclear structure research group has been actively involved in research at the High Flux Beam Reactor, Brookhaven National Laboratory, since 1980 when the TRISTAN on-line isotope separator was installed at BNL. During the period 1980-86 TRISTAN operated as a facility which provided beam time and support services to independent user groups. Since then the format has evolved into a collaborative effort involving personnel from Brookhaven National Laboratory, Clark University, and other institutions both in the U.S. and from abroad. The scope of our initiatives has broadened to include the tailored beam facility at BNL and joint projects with scientists at other facilities, most notably the Institute Laue-Langevin, Grenoble, France, and the ATLAS accelerator, Argonne National Laboratory.

From the scientific perspective the major impact of our collaborative program has been the focussing of effort on several research themes deemed to have critical interest by the group. Currently, four themes (1) the crucial effects of the proton-neutron interaction on the evolution of nuclear structure and its relation to collectivity, (2) the characterization of nuclei far-from-stability accessible only with radioactive ion beams (RIBs), (3) the appearance and role of dynamical symmetries in nuclei and the characterization of transitions among these limits, and (4) the characterization and prediction of properties of nuclei far-from-stability of importance to astrophysical mechanisms of nucleosynthesis. The focussing of the research effort on these four themes is evident in the progress reported herein and in recent publications involving Clark personnel.

In preparation for the advent of a first generation radioactive beam facility at Oak Ridge National Laboratory, a transportable coulomb excitation system is being designed and constructed at BNL for use in studies of low lying states of radioactive beam nuclei. It is envisioned that this 
apparatus will be used at other radioactive beam facilities as these become available throughout the world. In addition, the feasibility of equipping the tailored neutron beam facility at the BNL High Flux Beam Reactor with an array of Compton-suppressed Ge detectors is being studied. This development will permit enhanced studies at high excitation energy of nuclei near stability. Clark University graduate students, Charles Barton and Brian Foy, are both active in the development of these new experimental tools.

Recently, we have collaborated with Cary Davids of Argonne National Laboratory and colleagues from the University of Maryland and Vanderbilt University in measurements of masses and the decay of nuclei near the proton drip line using the Fragment Mass Analyzer (FMA) at the ATLAS accelerator facility. Future experiments at ATLAS are anticipated.

These projects and other progress are described in more detail below.

\section{B . Publications}

During the period, August 1, 1993 - July 31, 1994, covered by this report, a number of projects were completed and submitted for publication. The following list includes papers published, accepted for publication, or under review at the present time.

\section{Letters}

F-Spin Purity of $2{ }_{1} \pm$ States in Even-Even Nuclei A. Wolf, O. Scholten, and R. F. Casten

Phys. Lett. 312B, 372-376 (1993).

A Simple Shell Model Interpretation of the Evolution of Structure in Pre-collective Nuclei R. F. Casten, N. V. Zamfir, and D. S. Brenner Phys. Lett. 324B, 267-272 (1994).

Evolution of Pre-collective Nuclei and a Tripartite Classification of Nuclear Structure N. V. Zamfir, R. F. Casten, and D. S. Brenner Phys. Rev. Lett. 72, 3480-3482 (1994).

Distribution of Low Lying Quadrupole Phonon Strength in Nuclei N. Pietralla, P. von Brentano, R. F. Casten, T. Otsuka, and N. V. Zamfir Phys. Rev. Lett., submitted. 
Empirical Global Anharmonic Vibrator Spectra and the IBA Model

N. V. Zamfir and R. F. Casten

Phys. Lett., submitted.

Statistical Investigations of Factors Affecting Rotational Motion in Even-Even Nuclei

Jing-ye Zhang, N. V. Zamfir, and R. F. Casten

Phys. Rev. Lett., submitted.

\section{Articles}

Absolute B(E1) Values in the Shape Transitional 148-152 Sm Isotopes

A. Jungclaus, H. G. Börner, J. Jolie, S. Ulbig, R. F. Casten, N. V. Zamfir, P. von Brentano, and K. P. Lieb.

Phys. Rev. C47, 1020-1026 (1993).

Relation of Collective Observables to Nuclear Shapes

J.-Y. Zhang, R. F. Casten, and N. V. Zamfir

Phys. Rev. C48, R10-R12 (1993).

g-Factor of the $3 / 2 \pm 93.6 \mathrm{keV}$ Level in $21 \mathrm{Sr}$

A. Wolf, R. L. Gill, D. S. Brenner, Z. Berant, R. B. Schuhmann, and N. V. Zamfir

Phys. Rev. C48, 562-565 (1993).

Lifetimes and Electromagnetic Decay Properties of Negative-Parity States in $150.152 .154 \mathrm{Sm}$ from $\left(\mathrm{n}, \mathrm{n}^{\prime} \gamma\right)$ Measurements

A. Jungclaus, T. Belgya, D. P. DiPrete, M. Villani, E. L. Johnson, E. M. Baum, C. A. McGrath, S. W. Yates, and N. V. Zamfir

Phys. Rev. C48, 1005-1009 (1993).

Semiempirical Determination of Effective p-n Monopole Matrix Elements

S. Pittel, P. Federman, G. E. Arenas Peris, R. F. Casten, and W.-T. Chou

Phys. Rev. C48, 1050-1055 (1993).

Qctupole Fragmentation and $\mathrm{O}(6)$ Symmetry in Even-A Pt Isotopes

N. V. Zamfir, P. D. Cottle, J. L. Johnson, and R. F. Casten

Phys. Rev. C48, 1745-1751 (1993).

Effective Valence Spaces and Collectivity in Neutron Deficient Dy-Pt Nuclei W.-T. Chou, R. F. Casten, R. L. Gill, N. V. Zamfir, and D. S. Brenner Int. J. Mod. Phys. E2, 821-826 (1993). 
Phenomenological Signature for the Onset of Strong Octupole Correlations

N. V. Zamfir, P. von Brentano, and R. F. Casten

Phys. Rev. C49, R605-R607 (1994).

Evidence for Phase Transitional Behavior of Even-Even Nuclei from Differential Observables A. Wolf, R. F. Casten, N. V. Zamfir, and D. S. Brenner

Phys. Rev. C49, 802-807 (1994).

Correlation between $\varepsilon / \Delta$ and the P Factor

B. D. Foy, R. F. Casten, N. V. Zamfir, and D. S. Brenner

Phys. Rev. C49, 1224-1226 (1994).

Robust Predictions of the Interacting Boson Approximation Method

R. F. Casten, P. von Brentano, and N. V. Zamfir

Phys. Rev. C49, 1940-1948 (1994).

Evidence for a Spherical Subshell at $N=164$

D. S. Brenner, N. V. Zamfir, and R. F. Casten

Phys. Rev. C50, 490-492 (1994).

Performance of Conventional Fast Photomultipliers in an External Magnetic Field

A. Wolf, R. L. Gill, D. S. Brenner, Z. Berant, and R. B. Schuhmann

Nucl. Instr. Meth., in press.

Extension of a Nearly Global Anharmonic Vibrator Description of Nuclei to Non-Yrast States

W.-T. Chou, R. F. Casten, N. V. Zamfir, D. S. Brenner, and D. Bucurescu

Nucl. Phys., in press.

Study of Low-Spin States in ${ }^{122} \mathrm{Cd}$

N. V. Zamfir, R. L. Gill, D. S. Brenner, R. F. Casten, and A. Wolf

Phys. Rev. C, submitted.

\section{Conference Proceedings}

Phenomenological Correlations in Nuclear Structure: An Opportunity for Nuclear Astrophysics and a Challenge to Theory

R. F. Casten and N. V. Zamfir

First Symposium on Nuclear Physics in the Universe, Oak Ridge National Laboratory, Oak Ridge, TN, September (1992), M. W. Guidry and M. R. Strayer, Eds., (Institute of Physics, Bristol, U.K., 1993) p. 413-431. 
Correlations in Nuclear Observables: Towards a Synthesis of Structural Evolution

R. F. Casten, N. V. Zamfir, and D. S. Brenner

8th International Symposium on Capture Gamma-ray Spectroscopy and Related Topics, Fribourg, Switzerland, September (1993), J. Kern, Ed., (World Scientific, Singapore, 1994) p. 29-42.

\section{F-Spin Purity of $2{ }_{1}+$ States in Even-Even Nuclei}

A. Wolf, O. Scholten, and R. F. Casten

8th International Symposium on Capture Gamma-ray Spectroscopy and Related Topics, Fribourg, Switzerland, September (1993), J. Kern, Ed., (World Scientific, Singapore, 1994) p. 473-475.

New Signatures for Phase/Shape Transitional Regions

N. V. Zamfir, R. F. Casten, J.-Y. Zhang, and D. S. Brenner

8th International Symposium on Capture Gamma-ray Spectroscopy and Related Topics, Fribourg, Switzerland, September (1993), J. Kern, Ed., (World Scientific, Singapore, 1994) p. 479-481.

Predicting Properties of Exotic Collective Nuclei Using Valence Nucleon Correlation Schemes D. S. Brenner

The Proceedings of the Third International Conference on Radioactive Nuclear Beams, East Lansing, MI, May (1993), D. J. Morrissey, Ed., (Editions Frontieres, Gif-sur-Yvette, France, 1994) p 219-223.

A New Simple Global Phenomenology of Nuclear Shape Transition

R. F. Casten, N. V. Zamfir, and D. S. Brenner

The Proceedings of the Third International Conference on Radioactive Nuclear Beams, East Lansing, MI, May (1993), D. J. Morrissey, Ed., (Editions Frontieres, Gif-sur-Yvette, France, 1994) p 229-233 .

Glohal Phenomenology of Mean Field Structure and Phase Transitional Behavior in Nuclei

N. V. Zamfir, R. F. Casten, and D. S. Brenner

XVII Nuclear Physics Symposium at Oaxtepec, Oaxtepec, Mexico, January (1994), in press.

Universal Correlations of Collective Observables: Empirical Phenomenology and Model Interpretations

R. F. Casten, N. V. Zamfir, and D. S. Brenner

International Symposium on Frontiers of Nuclear Structure Physics, Tokyo, Japan, March (1994), in press. 
Correlations of Collective Ohservables and the Phonon Structure of Nuclei

R. F. Casten and N. V. Zamfir

International Conference on Nuclear Physics in the Late Nineties, Hanoi, Vietnam, March (1994), in press.

Evidence for Pervasive Phonon Structure of Nuclear Excitations

R. F. Casten and N. V. Zamfir

International Symposium in Honor of S. Belyaev, Philadelphia, PA, May (1994), in press.

E1 and E3 Transition Rates in the sdf-IBA

N. V. Zamfir

International Conference on Perspectives for the IBA on the Occasion of Its 20th Anniversary, Padova, Italy, June (1994), in press.

\section{Professional Activities}

In addition to formal publication, the results of our work have been presented orally, as posters and in abstracted form at professional meetings.

\section{Contributed Papers/Abstracts/Posters}

Qctupole Fragmentation Anomaly in Even-A Pt Isotopes

P. D. Cottle, J. L. Johnson, M. A. Kennedy, K. A. Stuckey, D. P. DiPrete, T. Belgya, E. M. Baum, E. L. Johnson, S. W. Yates, and N. V. Zamfir

Bull. Am. Phys. Soc. 38, 1832 (1993).

A Simple Shell Model Interpretation of the Evolution of Structure in "Pre-collective" Nuclei

N. V. Zamfir and R. F. Casten

Bull. Am. Phys. Soc. 38, 1845 (1993).

Universal Anharmonic Vibrator Description of Nuclei and Critical Phase Transition Behavior

D.S. Brenner, N.V. Zamfir, and R.F. Casten

Bull. Am. Phys. Soc. 38, 1845 (1993). 


\section{Invited Talks}

\section{D.S. Brenner}

The Structure of the Nucleus

South Hadley High School, South Hadley, MA, February (1994)

\section{N. V. Zamfir}

Low Lying Spectra of $\gamma$-Soft Nuclei

Workshop on High Spins and Novel Deformations, Trento, Italy, December (1993)

Global Phenomenology of Mean Field Structure and Phase Transitional Behavior in Nuclei XVII Nuclear Physics Symposium at Oaxtepec, Oaxtepec, Mexico, January (1994)

Phenomenolgical Constraints on the IBA Parameters for Negative-parity States

International Conference on Perspectives for the Interacting Boson Model on the Occasion of Its 20th Anniversary, Padova, Italy, June (1994)

Evidence for Pervasive Phonon Structure of Nuclear Excitations

International Symposium in Honor of S. Belyaev, Philadelphia, PA, May (1994)

E1 and E3 Transition Rates in the sdf-IBA

International Conference on Perspectives for the IBA on the Occasion of Its 20th Anniversary, Padova, Italy, June (1994)

\section{Education}

Each summer since 1989 D.S. Brenner has presented a series of lectures on nuclear chemistry and physics to students in the BNL Summer School on Nuclear Chemistry. This program, which is designed to provide intense and rigorous training in nuclear and radiochemistry to students who are between their sophomore and junior years in college, is focussed on addressing the shortfall in trained personnel for applied and basic research in nuclear science and medicine. Primary funding for the Summer School is provided by DOE with ancillary support 
from the Division of Nuclear Chemistry and Technology of the American Chemical Society and from Brookhaven National Laboratory.

\section{Professional Service}

D. S. Brenner organized and chaired a Symposium in Honor of Thomas T. Sugihara for the American Chemical Society 206th National Meeting held in Chicago, IL, August, 1993. He has reviewed manuscripts for Physical Review Letters, Physical Review C, and the International Journal of Modern Physics E as well as research proposals for DOE and for the Scientific and Engineering Research Council of Canada.

\section{Recent Initiatives}

In this section we include brief reports of recent research which is still in progress or which has been submitted for publication, but has not yet appeared in the open literature.

\section{1. g-Factor of the $3 / 2+121.8 \mathrm{keV}$ Level in ${ }^{99} \mathrm{Z} \mathrm{r}$}

\section{(Clark/BNL/Negev)}

The $\mathrm{g}$-factor of the first excited, $3 / 2^{+}, 121.8 \mathrm{keV}$ level of ${ }^{99} \mathrm{Zr}\left(\mathrm{t}_{1 / 2}=1.07 \mathrm{~ns}\right)$ has been measured using a beam of mass-separated fission products at the TRISTAN on-line isotope separator and the Integral Perturbed Angular Correlation method. The $\mathrm{A}=99$ fission products, containing the short-lived $\left(t_{1 / 2}=1.5 \mathrm{~s}\right){ }^{99} \mathrm{Y}$ which decays to ${ }^{99} \mathrm{Zr}$, were deposited on an aluminized tape and transported periodically to the center of a superconducting magnet (maximum field: 6.3 Tesla). Four HpGe detectors, which were placed concentrically around the magnet at $9.5 \mathrm{~cm}$ from its center were used to measure the correlation. The angles between the detectors were $90^{\circ}(1)$, 
$120^{\circ}(2)$ and $150^{\circ}(3)$, where the numbers in parentheses indicate the number of detector pairs at the given angle. The data were accumulated in event mode and sorted off-line using a $\mu$-Vax computer. In a ten day run approximately $10^{7}$ coincidence events were recorded for each field direction. In a separate experiment, nonperturbed angular correlations were measured using the same $\mathrm{HpGe}$ detectors, in order to determine the anisotropous of the $\gamma-\gamma$ correlations.

Our analysis of the data yields an experimental g-factor for the $121.8 \mathrm{keV} 3 / 2+$ level in ${ }^{99} \mathrm{Zr}$ of +0.29 (4). This result can be compared favorably to a theoretical calculation by Kisslinger and Sorensen [1] for an almost pure $d_{3 / 2}$ single neutron quasiparticle state for the $\mathrm{N}=59$ isotone ${ }^{107} \mathrm{Cd}$ which gives $g_{\text {theory }, 3 / 2^{+}}=+0.25$ for $g_{R}=Z / A$ [1]. Alternatively, the core-excitation model of deShalit [2] can be used to estimate the $\mathrm{g}_{3 / 2^{+}}$for ${ }^{99} \mathrm{Zr}$ and yields $+0.56(10)$ in disagreement with our experimental value. Thus, we conclude that the good agreement found with the KisslingerSorensen model, which is intended for spherical nuclei, provides evidence that the $3 / 2+121.8 \mathrm{keV}$ level in ${ }^{99} \mathrm{Zr}$ is indeed a spherical state.

The results of this work will be submitted for publication in the near future.

[1] L. S. Kisslinger and R. A. Sorensen, Rev. Mod. Phys. 35, 853 (1963).

[2] A. deShalit, Phys. Rev. 122, 1530 (1961).

2. Evidence for a Spherical Subshell at $N=164$ (Clark/BNL/Bucharest-Magurele)

The evolution from spherical, closed shell structures to stable deformed shapes in nuclei has been understood as a competition between two attractive pairwise interactions, the spherical- 
driving, pairing interaction between like particles and the deformation-driving, quadrupole protonneutron interaction. The effect of the latter is manifested in the quadrupole deformation, $\varepsilon$, which can be deduced from measurements of the reduced transition probability between the ground and first excited $2^{+}$state of an even-even nucleus. The former is measured by the pairing gap parameter, $\Delta$, which is calculated from empirical mass differences between adjacent nuclei with odd and even numbers of nucleons. The ratio of these two quantities, $\varepsilon / \Delta$, has often been used to characterize the transition from spherical to deformed shapes. While this simple formulation has proved to be a sensitive test of structure, the experimental quantities needed can be difficult to measure accurately and are not well known for many nuclei, especially those far from stability.

Recently, the simple factor $P \equiv N_{p} N_{n} /\left(N_{p}+N_{n}\right)$ has been shown to successfully systemize mean field observables and to reproduce the systematic variation of $\varepsilon / \Delta$ with $\mathrm{A}$. This is illustrated in Fig. 1 where the global systematics of $\varepsilon / \Delta$ and $P$ are compared. Here $N_{p}$ and $N_{n}$ are the numbers of valence protons and neutrons, counted as particles or holes from the nearest spherical shell closure and thus $\mathrm{P}$ depends directly on shell and subshell boundaries. For example, good agreement between the $\varepsilon / \Delta$ and P plots in the A 100 region is only achieved if the well-known subshells at $Z=40$ and 64 are taken into account in the calculation of $P$. This analysis, when extended to the actinide region, provides evidence for a possible shell or subshell at $\mathrm{N}=164$.

Fig. 2 shows a comparison between the $\varepsilon / \Delta$ systematics and those of $P$ for the actinide region beyond A 200 which serves to illustrate the usefulness of this kind of global correlation. Comparison of parts (a) and (b) shows that the values for $\varepsilon / \Delta$ peak near A 240 reaching a level of $\sim 0.40$ which is comparable to that found for known nuclei in the rare earth region. In contrast, 

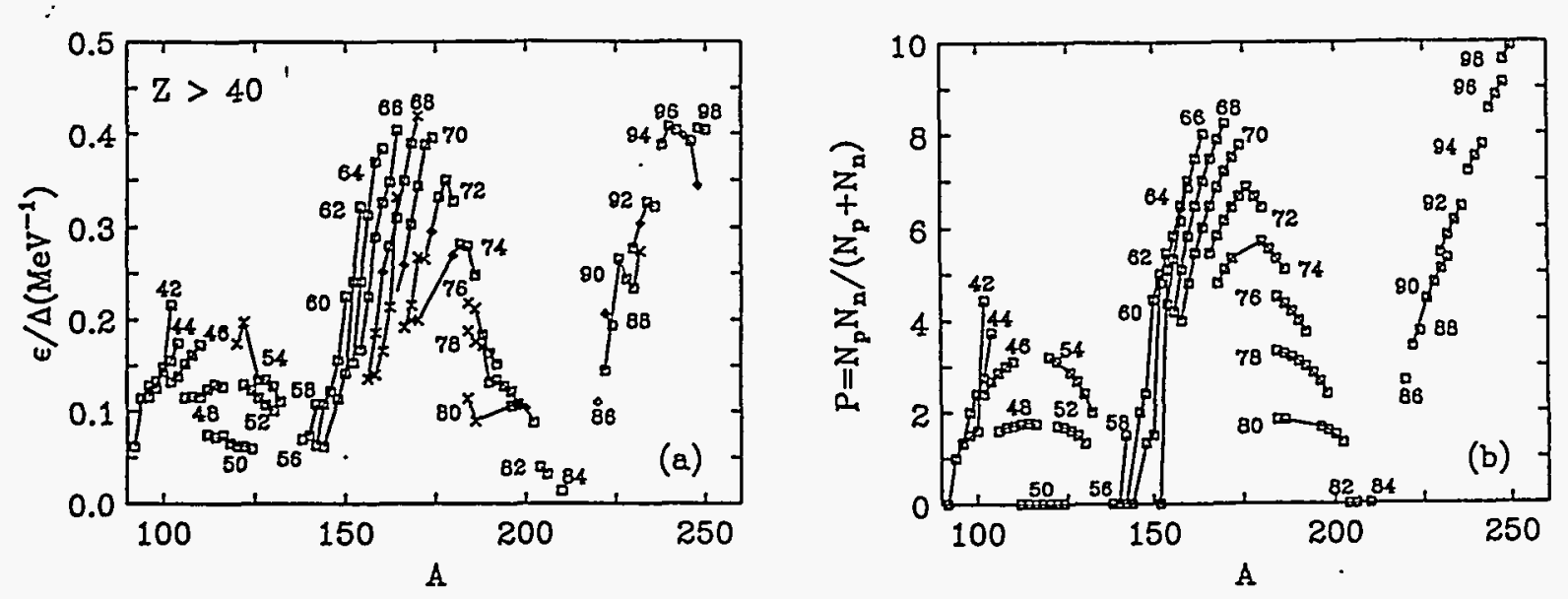

Fig. 1. Empirical $\varepsilon / \Delta$ values and calculated $P$ factors plotted against the mass number $A$.

values of $P$ for actinide nuclei calculated using classical shell assumptions $(Z=82,126$ and $N=$ $126,184)$ rise above the maximum of the rare earth peak and will continue to rise until mid-shell nuclei are reached. This discrepancy between the $\varepsilon / \Delta$ and $\mathrm{P}$ systematics can be resolved, however, simply by invoking a significant spherical neutron shell gap at some value of $\mathrm{N}$ less than 184 . Examination of a traditional Nilsson diagram for this region suggests that such a spherical shell gap at $\mathrm{N}=164$, above the $\mathrm{g}_{9 / 2}, \mathrm{i}_{11 / 2}$ and $\mathrm{j}_{15 / 2}$ orbits is a distinct possibility. The result of introducing the $N=164$ closure is shown in part (c) of Fig. 2. Clearly a closure at $N=164$ significantly improves the agreement between the two systematics in the actinide region. The relative height of the actinide peak, its location and the rollover of the isotopic chains are closely simulated in the $\mathrm{P}$ plot. Therefore, it seems reasonable to propose that the $\varepsilon / \Delta$ systematics are indicative of a neutron shell or subshell closure and that this most likely arises from a gap below the $d_{5 / 2}$ spherical orbit. A shell or subshell at $\mathrm{N}=164$ will likely give rise to enhanced stability for superheavy elements and should guide future searches for these elusive nuclei. 

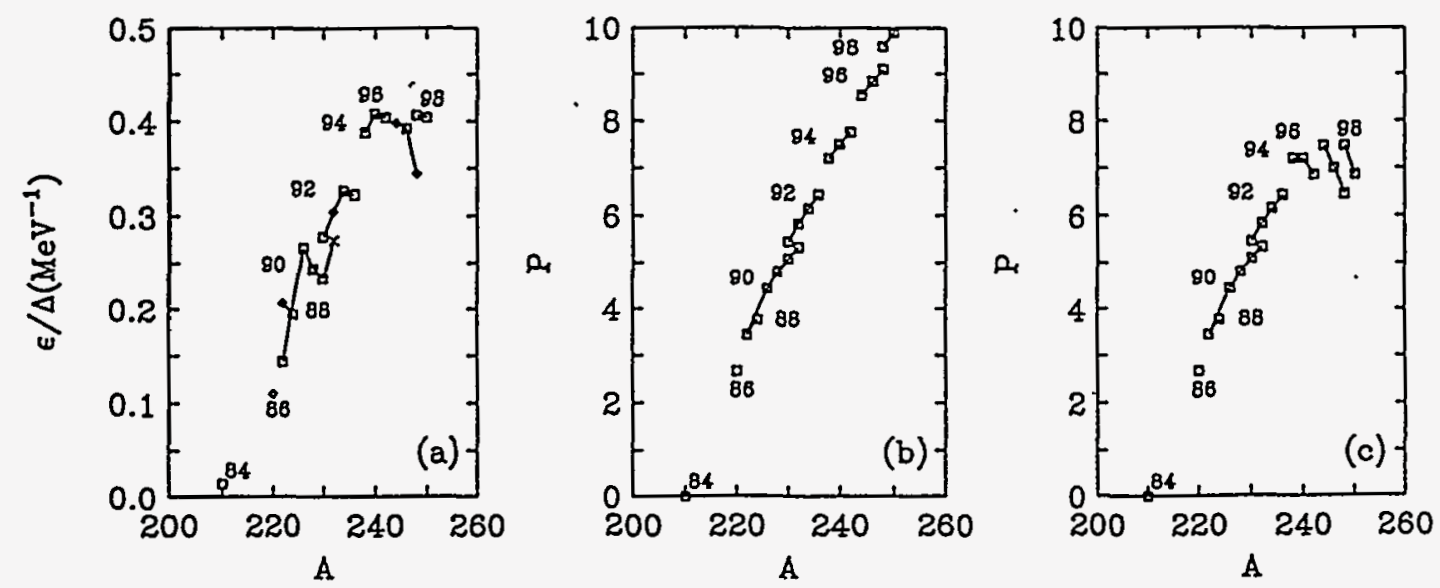

Fig. 2. (a) $\varepsilon / \Delta$ vs. A for the actinide region; (b) $P$ vs. A for the same nuclei as in panel calculated assuming shell closures at $\mathrm{Z}=82,126$ and $\mathrm{N}=126,184$; (c) $\mathrm{P}$ vs. $\mathrm{A}$ where is $\mathrm{P}$ is calculated assuming a subshell closure at $\mathrm{N}=164$.

A full discussion of these systematics and that of additional experimental observables has recently appeared in the Physical Review.

\section{The Phonon Structure of ${ }^{122} \mathrm{Cd}$}

\section{(Clark/BNL/Bucharest-Magurele)}

The Cadmium nuclei, with proton number near the magic number 50, present interesting aspects of nuclear structure, namely the coexistence and mixing of vibrational multiphonon degrees of freedom, such as those identified by our group in ${ }^{114} \mathrm{Cd}$, with intruder rotational structures which arise from the promotion of a proton pair across the $Z=50$ shell gap. The excitation energy of the intruder configuration depends on the size of the shell gap in comparison to the strength of the integrated residual proton-neutron interaction, and it depends directly on the number of active neutrons. 
The present work is part of a systematic study of neutron rich Cd isotopes produced in fission of ${ }^{235} \mathrm{U}$ at the TRISTAN on-line mass separator facility. The ${ }^{122} \mathrm{Cd}$ measurements

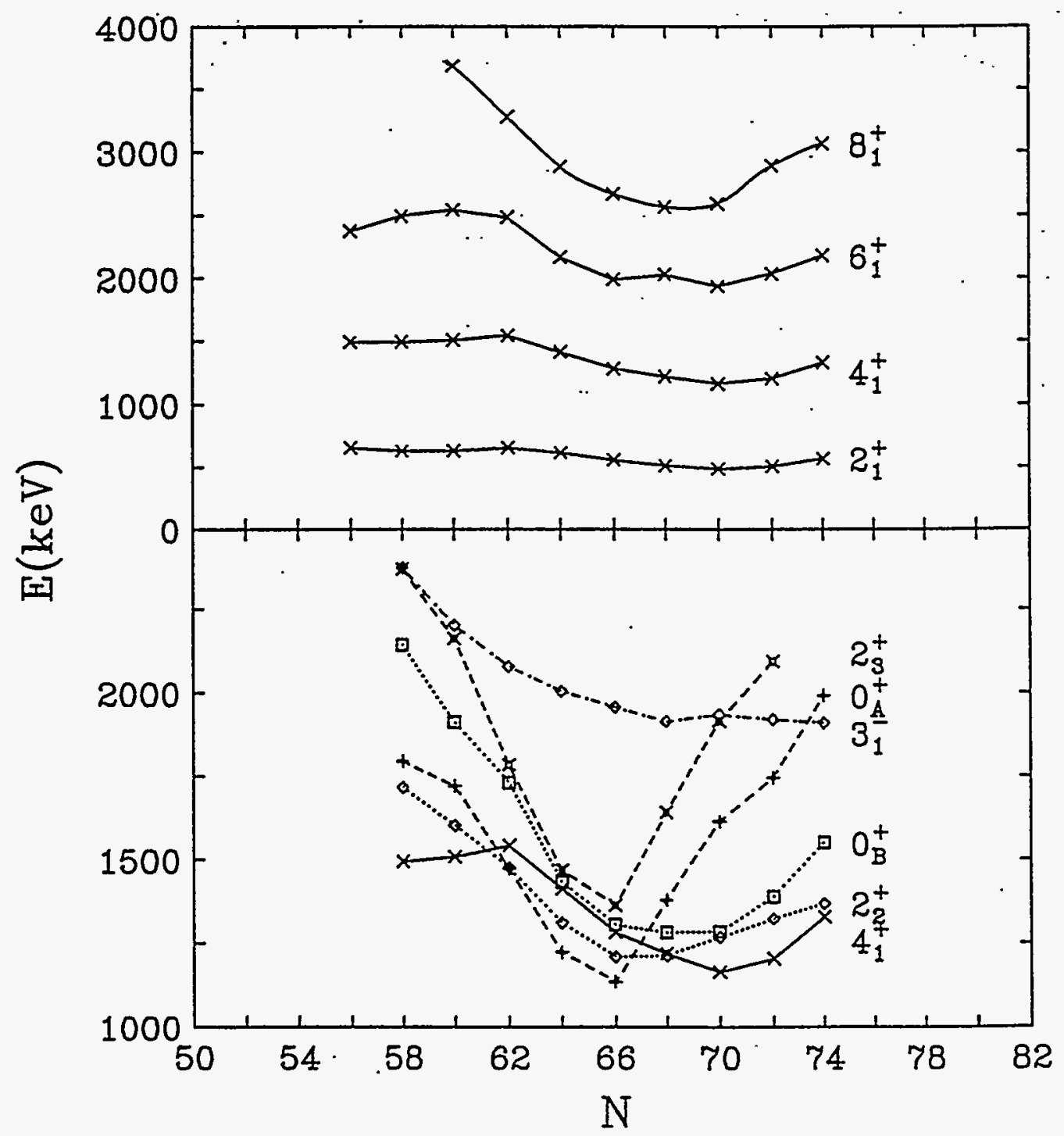

Fig. 3. Energy systematics of the cadmium nuclei.

included $\gamma$-ray singles, $\gamma-\gamma$ coincidence, $\gamma-\gamma$ angular correlation and $\beta-\gamma-\gamma$ coincidence spectroscopy. The $\gamma$-ray spectra of ${ }^{122} \mathrm{Ag}$ decay were measured by collecting the $\mathrm{A}=122$ beam 
from TRISTAN on a movable aluminized-mylar tape while simultaneously counting for $3 \mathrm{~s}$. Subsequently the tape was moved to minimize $\gamma$ activity from other isobars.

From these comprehensive measurements we have been able to characterize much of the lowlying structure of ${ }^{122} \mathrm{Cd}$. Among our new results are the establishment of the yrast band up to spin $8^{+}$, unambiguous identification of the $\mathrm{O}_{2}{ }^{+}$and $\mathrm{O}_{3}{ }^{+}$states, measurement of the lifetime of the $2_{1}{ }^{+}$ level and a tentative assignment of the $3_{1}{ }^{-}$state. These results serve to extend the systematics of the $C d$ isotopes very far from stability and across a very broad range of the $\mathrm{N}=50-82$ shell (see Fig. 3 for a plot of the level systematics for neutron-rich Cd nuclei). This, in turn, provides a "laboratory" where the 2 particle- 2 hole intruder configuration can be studied within the context of the multiphonon structures of most low lying states in Cd nuclei. At the present juncture the nature of the $\mathrm{O}_{2}{ }^{+}$and $\mathrm{O}_{3}{ }^{+}$states remains vexing and unresolved for the $\mathrm{Cd}$ isotopes including ${ }^{122} \mathrm{Cd}$, where the fact that both the $\mathrm{O}_{2}+$ and $\mathrm{O}_{3}{ }^{+}$states decay to the $2{ }_{1}{ }^{+}$level suggests that both states may have substantial 2-phonon character.

The results of this work have been submitted for publication.

\section{Mass and Decay of the $\mathrm{N}=\mathrm{Z}$ Nuclide ${ }^{68} \mathrm{Se}$}

(Argonne/Vanderbilt/Maryland/Clark)

The $\mathrm{N}=\mathrm{Z}$ nucleus ${ }^{68} \mathrm{Se}$ may be produced in stars during explosive Si-burning as part of a series of $(\alpha, \gamma)$ reactions beginning with ${ }^{56} \mathrm{Ni}(\alpha, \gamma)$. For nuclei with $\mathrm{Z}>28$ high Coulomb barriers prevent $\alpha$-capture reactions from taking place except at the extreme temperatures found in Type I Supernova. Under these conditions there is an equilibrium established between $(\alpha, \gamma)$ capture and the inverse $(\gamma, \alpha)$ photodistintegration reactions and the ${ }^{68} \mathrm{Se} \mathrm{Q}$-value appears in the argument of an 
exponential expression for the rate equation. Thus, even a relatively crude mass measurement for ${ }^{68} \mathrm{Se}$ can determine whether the $(\alpha, \gamma)$ reaction chain can proceed further. Previous work [1] on the next lightest $\alpha$-particle nucleus, ${ }^{64} \mathrm{Ge}$, has shown that it can be produced in this fashion, explaining much of the natural abundance of ${ }^{64} \mathrm{Zn}$.

We recently joined in a successful experiment to produce and study the decay of ${ }^{68} \mathrm{Se}$ using the FMA at ATLAS. ${ }^{68} \mathrm{Se}$ was produced in inverse kinetics using the ${ }^{12} \mathrm{C}\left({ }^{58} \mathrm{Ni}, 2 \mathrm{n}\right)$ reaction at 200 $\mathrm{MeV} .{ }^{68} \mathrm{Se}$ ions were collected on an aluminized tape at the focal plane of the FMA and transported to a position in front of various detector pairs. Plastic scintillation( $(\beta)-G e(\gamma)$ and LEPS $\mathrm{Ge}(\gamma)-\mathrm{Ge}(\gamma)$ coincidence spectra were recorded as a function of time following bombardment for $10 \mathrm{~s}$ intervals. Preliminary analysis of the data confirms the level scheme of the daughter nucleus, ${ }^{68} \mathrm{As}$, proposed by P. Baumann et al. [2]. Detailed analysis of the data is underway and is expected to yield a value for the mass of ${ }^{68} \mathrm{Se}$ with an error in the $100 \mathrm{keV}$ range.

[1] C. N. Davids and D. R. Goosman, Phys. Rev. C7, 122 (1973).

[2] P. Baumann et al., Proceedings of the Workshop on Nuclear Structure of Light Nuclei Far From Stability, Ed. G. Klotz, Obernai, France (1989) p 43.

\section{E. Personnel}

Dr. Daeg S. Brenner, Professor of Chemistry, Adjunct Professor of Physics Clark University

Dr. Nicolae V. Zamfir, Consultant, Clark University (4/92- )

Dr. Alex Wolf, Senior Research Associate, Clark University (8/92-8/93)

Mr. Charles Barton, Graduate Student, Physics Department, Clark University

Mr. Brian Foy, Graduate Student, Chemistry Department, Clark University 
D. S. Brenner is principal investigator for this project. N. V. Zamfir, a visiting scholar from the Institute of Atomic Physics, Bucharest-Margurele, Romania, receives joint support from BNL and Clark University. A. Wolf, a distinguished research physicist from the Negev Research Centre, Israel, recently completed a very productive year working in our group at BNL. Charles Barton and Brian Foy are Clark University graduate students who receive support from our DOE grant. Barton is in residence at BNL and Foy supports our activities at Clark University.

\section{ACKNOWLEDGEMENT}

We acknowledge the U.S. Department of Energy (Grant No. DE-FG02-88ER40417) for the primary support of our nuclear structure research program. The support of the U.S. Department of Energy for the Neutron Nuclear Physics Group at Brookhaven National Laboratory, and for our collaborators at various universities and laboratories is also recognized and appreciated.

We also wish to thank our many colleagues with whom we have collaborated during the past $\rightarrow$

year and especially Ron Gill, Dean MacDonald and Rick Wall at TRISTAN for their kind (i)

hospitality. Finally, we acknowledge the cheerful and excellent clerical support provided by Jackie Mooney at BNL. 